\begin{tabular}{|c|c|c|}
\hline $\begin{array}{l}\text { ITC 3/47 } \\
\text { Journal of Information Technology }\end{array}$ & \multicolumn{2}{|c|}{$\begin{array}{c}\text { A New Hybrid Genetic Algorithm for the Grey Pattern } \\
\text { Quadratic Assignment Problem }\end{array}$} \\
\hline $\begin{array}{l}\text { Vol. 47/ No. } 3 / 2018 \\
\text { pp. 503-520 }\end{array}$ & Received 2018/05/02 & Accepted after revision 2018/07/16 \\
\hline $\begin{array}{l}\text { DO1 10.5755/J01.tc.47.3.20728 } \\
\text { (9) Kaunas University of Technology }\end{array}$ & \multicolumn{2}{|c|}{ ressef http://dx.doi.org/10.5755/j01.itc.47.3.20728 } \\
\hline
\end{tabular}

\title{
A New Hybrid Genetic Algorithm for the Grey Pattern Quadratic Assignment Problem
}

\section{Alfonsas Misevičius}

Kaunas University of Technology, Department of Multimedia Engineering, Studentu st. 50-416a/400, LT-51368 Kaunas, Lithuania, tel. +370-37-300372, alfonsas.misevicius@ktu.lt

\section{Evelina Stanevičienè}

Kaunas University of Technology, Department of Multimedia Engineering, Studentų st. 50-416a/408, LT-51368 Kaunas, Lithuania, tel.+370-37-300373, evelina.staneviciene@ktu.lt

Corresponding author: alfonsas.misevicius@ktu.lt

In this paper, we propose an improved hybrid genetic algorithm for the solution of the grey pattern quadratic assignment problem (GP-QAP). The novelty is the hybridization of the genetic algorithm with the so-called hierarchical iterated tabu search algorithm. Very fast exploration of the neighbouring solutions within the tabu search algorithm is used. In addition, a smart combination of the tabu search and adaptive perturbation is adopted, which enables a good balance between diversification and intensification during the iterative optimization process. The results from the experiments with the GP-QAP instances show that our algorithm is superior to other heuristic algorithms. Many best known solutions have been discovered for the large-scaled GP-QAP instances.

KEYWORDS: computational intelligence, heuristics, hybrid genetic algorithms, tabu search, combinatorial optimization, grey pattern quadratic assignment problem.

\section{Introduction}

The grey pattern quadratic assignment problem (GP$\mathrm{QAP})$ is a special case of the well-known combinatorial optimization problem, the quadratic assignment problem (QAP) [2]. GP-QAP can be formulated as follows [15]. Given two matrices $\boldsymbol{A}=\left(a_{i j}\right)_{n \times n}$ and $\boldsymbol{B}=\left(b_{k l}\right)_{n \times n}$ and the set $\Pi_{n}$ of permutations of the integers from 1 to $n$, find a permutation $p \in \Pi_{n}$ that minimizes

$$
z(p)=\sum_{i=1}^{n} \sum_{j=1}^{n} a_{i j} b_{p(i) p(j)}
$$


where $a_{i j}=1$ for $i, j=1, \ldots, m(1 \leq m<n)^{1}$ and $a_{i j}=0$ otherwise. The values of the matrix $\left(b_{k l}\right)_{n \times n}$ may be seen as distances between every pair of $n$ objects (elements), $b_{k l}=b_{l k}, b_{k k}=0, k, l=1, \ldots, n$. In the context of the GPQAP, the values $b_{k l}$ are defined according to the following rule [15]:

$$
\begin{aligned}
& b_{k l}=b_{(r-1) n_{2}+s(t-1) n_{2}+u}=\omega_{r s t u}, \omega_{r s t u}= \\
& \max _{w_{1}, w_{2} \in\{-1,0,1\}}\left\{1 /\left(\left(r-t+w_{1} n_{1}\right)^{2}+\left(r-t+w_{2} n_{2}\right)^{2}\right)\right\}
\end{aligned}
$$

where $r, t=1, \ldots, n_{1}, s, u=1, \ldots, n_{2}, n_{1} \times n_{2}=n$. The interpretation of the quantity $\omega_{\text {rstu }}$ is as follows (see also [15]). We may consider $m$ electrons that have to be put on grid's squares. Then, $\omega_{\text {rstu }}$ may be thought of as a quantity proportional to repulsion force between two electrons $i$ and $j(i, j=1, \ldots, n)$ located in the grid positions $k=p(i)$ and $l=p(j)$ with the coordinates $(r, s)$ and $(t, u)$. The electrons are to be arranged in such a way that the sum of the intensities of the repulsion forces is minimized.

So, there is a grid of dimensions $n_{1}$ by $n_{2}$. In the grid, there are $n=n_{1} \times n$ squares: there are $m$ black squares while the rest of the squares are white. This forms a grey pattern of density $m / n$. We seek to have a grey pattern where the black points are distributed in the most uniform possible way along the grid.

According to the above formulation, $p$ denotes a permutation and $p(i), p(j)$ denote the corresponding items (elements) of the permutation. The first $m$ items of every feasible permutation may be considered as a solution of the GP-QAP. In this way, the objective is to find the best available, optimal solution, i.e., the permutation items $p(1), \ldots, p(m)(1 \leq p(i) \leq n$, $i=1, \ldots, m)$ such that the sum $\sum_{i=1}^{m} \sum_{j=1}^{m} b_{p(i) p(j)}$ (considered as an objective function of the GP-QAP) is as minimal as possible, that is:

$$
z(p)=\sum_{i=1}^{m} \sum_{j=1}^{m} b_{p(i) p(j)} \rightarrow \text { minimum }
$$

In formulation (3), only the matrix $\boldsymbol{B}$ and the values of $n, m$ are necessary; meanwhile, the matrix $\boldsymbol{A}$ is not needed at all. In our work, we use this formulation, rather than the general formulation (1). Also, note

\footnotetext{
1 In our work, we will consider $m \leq n / 2$.
}

that the GP-QAP-solution (the elements $p(1), \ldots, \mathrm{p}(m)$ ) can be associated with an $m$-(sub)set $M$ such that $M=$ $\{p(i): i=1, \ldots, m\},|M|=m$ (see [5]). Analogously, the elements $p(m+1), \ldots, p(n)$ can be related to an $n-m$-(sub) set $N$, where $N=\{p(i): i=m+1, \ldots, n\},|\mathrm{N}|=n-m$.

Exchanging two black (or two white) squares with each other does not change the value of the objective function, thus many permutations with the same objective value, $z(p)$, may exist. So, there exist at least $m$ ! optimal solutions.

There can be other contexts of the problem defined by formula (1). For example, we can consider $n$ points in the plane or $n$ nodes of a network and we then may wish to find a cluster of $m$ points, which minimizes the total distance between all pairs of points in the cluster. This cluster can be interpreted as the tightest cluster of $m$ points [5]. This is similar to the max-cover problem [3] where one wishes to find the location of several facilities which cover the maximum number of points.

The other example of applications includes the selection of a group of $m$ people out of $n$ available people [5]. The distance between a pair of persons is a measure of compatibility (a measure of the ability to work together). The ideal group will have the most mutual compatibility and the least potential for tension.

For the solution of the GP-QAP, the computational intelligence approaches are well applicable, including the exact and heuristic algorithms. The exact algorithms are suited only for small-sized problems [5, 6]. For larger problem instances, heuristic algorithms are used: single-solution-based algorithms (local search, tabu search $[5,15])$, population-based algorithms (genetic/evolutionary algorithms $[5,15,16]$. Among heuristic algorithms, hybrid genetic/evolutionary algorithms have been shown to be very effective $[5,11,12,14]$.

In this paper, we are attempting to further improve the performance of hybrid genetic algorithms by proposing some more new enhancements ${ }^{2}$. The main contributions are as follows.

1 The so-called hierarchical iterated tabu search (ITS) algorithm and its hybridization with the genetic algorithm are applied to the grey pattern quadratic assignment problem for the first time.

2 We assume that the reader is familiar with the most basic concepts of the genetic algorithms [9] and also the local searchbased, tabu search-based heuristic algorithms $[1,8]$. 
2 Very fast evaluation of the neighbouring solutions within the tabu search algorithm is used.

3 Smart combination of the tabu search and greedy adaptive perturbation is adopted. This enables to achieve the beneficial synergy of diversification and intensification during the iterative optimization process.

The paper is organized as follows. In Section 1, some preliminaries are given. In Section 2, we describe a novel hybrid genetic algorithm for the grey pattern quadratic assignment problem. The results of the computational experiments with the proposed algorithm are presented in Section 3. The paper is completed with concluding remarks.

\section{Preliminaries}

A neighbourhood function $\Theta: \Pi_{n} \rightarrow 2^{\Pi_{n}}$ assigns for each $p \in \Pi_{n}$ a set $\Theta(p) \subseteq \Pi_{n}$ - the set of neighbouring solutions of $p$. With the permutation-based problems, a common practice is to use the 2-exchange neighbourhood function $\Theta_{2}$ which is defined in the following way: $\Theta_{2}(p)=\left\{p^{\prime}: p^{\prime} \in \Pi_{n}, \delta_{H}\left(p, p^{\prime}\right)=2\right\}$, where $\delta_{H}\left(p, p^{\prime}\right)$ is the Hamming distance between the permutations $p$ and $p^{\prime}$. (Remind that the Hamming distance between two permutations $p_{1}$ and $p_{2}$ can be declared as $\delta_{H}\left(p_{1}, p_{2}\right)=\left|\left\{i: p_{1}(i) \neq p_{2}(i)\right\}\right|$.) However, the order of the elements $p(1), \ldots, p(m)$ is not important in the GP-QAP, so we have to formulate the neighbourhood function in a more appropriate way. The 1-interchange neighbourhood function $\Theta_{1}$ is defined such that every neighbouring solution $p^{\prime} \in$ $\Theta_{1}(p)$ is obtained from the current solution $p$ by simply interchanging one element of $\{p(i): i=1, \ldots, m\}$ with another element of $\{p(j): j=m+1, \ldots, n\}$. Clearly, this neighbourhood function maintains solution feasibility, i.e., $\forall p \in \Pi_{n}: p^{\prime} \in \Theta_{1}(p) \Longrightarrow p^{\prime} \in \Pi_{n}$. More formally:

$$
\Theta_{1}(p)=\left\{p^{\prime}: p^{\prime} \in \Pi_{n}, \delta\left(p, p^{\prime}\right)=1\right\}
$$

where $\delta$ denotes the distance between solutions. The distance between two GP-QAP-solutions $p_{1}$ and $p_{2}$ can be defined in the following way:

$\delta\left(p_{1}, p_{2}\right)=m-\left|\left\{p_{1}(i): i=1, \ldots, m\right\} \cap\left\{p_{2}(i): i=1, \ldots, m\right\}\right|$
It can be seen that $0 \leq \delta \leq m, \delta(p, p)=0, \delta\left(p_{1}, p_{2}\right)=$ $\delta\left(p_{2}, p_{1}\right)$.

To be more precise, let $p(v)(u=1, \ldots, m)$ and $p(w)$ $(v=m+1, \ldots, n)$ be two items to be swapped. Then, a short notation of the form $p^{v, w}$ can be used such that $p^{v, w}(i)=\left\{\begin{array}{l}p(i), \quad i \neq v, w \\ p(v), \quad i=w . \\ p(w), i=v\end{array}\right.$. This means that $p^{v, w}$ is obtained from $p$ by interchanging the items $p(v)$ and $p(w)$ ( $p$ is said to move to $\left.p^{v, w}\right)$. Of course, $\delta\left(p, p^{v, w}\right)=$ $1, p^{v, v}=p,\left(p^{v, w}\right)^{v, w}=p$. It is of high importance to efficiently calculate the difference in the objective values when interchanging the items $p(v)$ and $p(w)$. The difference is calculated in $O(1)$ time by this formula:

$$
\begin{aligned}
& \Delta\left(p^{v, w}, p\right)=z\left(p^{v, w}\right)-z(p)= \\
& =2\left(c(p(w))-c(p(v))-b_{p(v) p(w)}\right)
\end{aligned}
$$

where $c(x)$ is a contribution of the element $x$ (the sum of related distances):

$$
c(x)=\sum_{y=1}^{m} b_{x p(y)}, x=1, \ldots, n
$$

After the exchange, the contributions are updated according to the expression:

$$
\begin{aligned}
& c(x)= \begin{cases}c(x)+b_{x p(v),} & x=p(w) \\
c(x)-b_{x p(w)}, & x=p(v) \\
c(x)+b_{x p(v)}-b_{x p(w)}, & x \neq p(v), x \neq p(w)\end{cases} \\
& x=1, \ldots, n
\end{aligned}
$$

The elements of the found solution determine the locations in the grid where the black squares have to be placed in. The coordinates $(r, s)$ of the black squares are derived according to these formulas: $r=\left\lfloor(p(i)-1) / n_{2}\right\rfloor+1, \quad s=\left((p(i)-1) \bmod n_{2}\right)+1$ (see also Figure 1 ).

Let us also introduce the concept of an opposition-based solution (opposite solution), which is in connection with what is known as an opposition-based learning (OBL) [17]. The rationale of opposition-based solutions is based on an assumption that it is more advantageous to consider an opposite solution with respect to the current solution from 
Figure 1

A graphical illustration of correspondence of the analytical solution to the graphical image

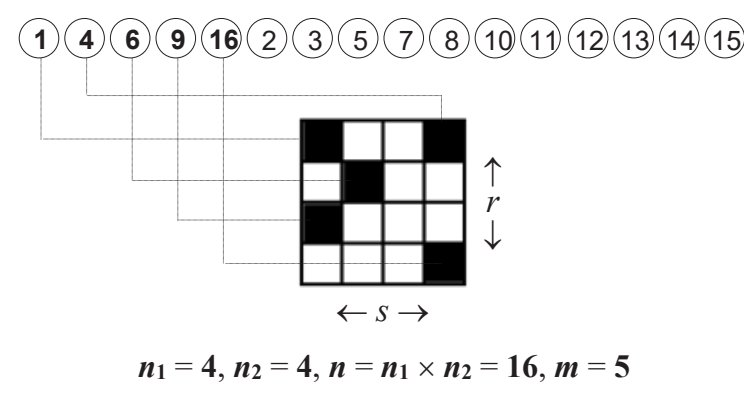

a search space, rather than a pure random solution generated in a blind random way. The helpfulness of using the opposition-based solutions has been confirmed by solving the maximum diversity problem (MDP) [19], which may be seen as a "sister problem" of the GP-QAP.

Definition 1. The GP-QAP-solution $\bar{p} \in \Pi_{n}$ is an opposition-based solution (opposite solution) with respect to the solution $p$ if $\delta(p, \bar{p})=m$.

Finally, we are defining a backbone solution (see also [19]).

Definition 2. The GP-QAP-solution $p^{\mathscr{B}} \in \Pi_{n}$ is a backbone solution (with respect to two underlying solutions $\left.p_{1}, p_{2}\right)$ if simultaneously $\delta\left(p^{\mathscr{Q}}, p_{1}\right) \leq\lceil m / 2\rceil$ and $\delta\left(p \circ, p_{2}\right) \leq\lceil m / 2\rceil$.

Roughly speaking, the backbone solution shares information with its both underlying solutions and is close enough to both of them (or possibly "equivalent" to the underlying solution(s) in the sense that $\delta\left(p^{\mathscr{\ell}}, p_{1}\right)=0$ and/or $\left.\delta\left(p^{\mathscr{Q}}, p_{2}\right)=0\right)$.

Within the genetic algorithm, the solution $p=$ $(p(1), \ldots p(m))$ is associated with an individual (in fact, the individual's chromosome). Then, the single element $p(i)$ corresponds to a gene of the current chromosome.

\section{Hybrid Genetic Algorithm for the Grey Pattern Quadratic Assignment Problem}

Our algorithm is based on the general hybrid genetic algorithm framework where the population-based evolutionary search (i.e., explorative search) is combined with the local improvement of the offspring (i.e., exploitative search) to enhance the overall search performance.

The hybrid genetic algorithm consists of six main components: a) creation of an initial (starting) population; b) parent selection; c) a crossover operator; d) an improvement of the produced offspring; e) updating of the population; and f) restart from the new population (if necessary). The high-level description of the hybrid genetic algorithm is presented in Figure 2.

The algorithm starts with creation of the initial population of fixed size $P S$ by paying attention to both the quality (fitness) of the population individuals and the mutual variability (i.e., mutual distance) between all the population members. The algorithm then performs iterations called generations until the pre-defined number of generations, $N_{\text {gen }}$, has been accomplished. At every generation, the standard genetic operations - selection, crossover, population replacement - take place (with the exception of mutation). The mutation procedure is integrated into the improvement algorithm - the hierarchical iterated tabu search (HITS). Our genetic algorithm also incorporates the restart mechanism in the cases of observed stagnation of the evolutionary process.

The components of the hybrid genetic algorithm are discussed in more detail in the subsequent sections.

\subsection{Initial Population Construction}

The initial population is generated as follows.

1 Let flag $=$ 'OFF', $P=\emptyset, k=1, l=1$. ( $l$ is the lexicographic index of the generated solution.)

2 If flag $=$ 'OFF', then generate a random permutation (solution) $p_{1}$; otherwise, generate an opposition-based random permutation $p_{l} . l=l+1$.

3 Apply the hierarchical iterated tabu search algorithm to the generated solution and get the improved solution $p_{l}^{*}$.

4 If ( flag $=$ 'OFF') and $(k=1)$, then: a) include the solution $p_{1}^{*}$ into the population $P$; b) flag = 'ON'; c) go to Step 2 .

5 If $(\text { flag }=\text { 'ON'})^{\prime}$ and $(k=1)$ and $\left(z\left(p_{l}^{*}\right)<\right.$ $z(p): p \in P$ ), then: a) replace the 1 st member of the population by the solution $\left.p_{l}^{*} ; b\right)$ go to Step 2 .

6 If $\left(\left(z\left(p_{l}^{*}\right) \neq z(p): \forall p \in P\right)\right.$ and $\left(\min _{p \in P}\left\{\delta\left(p_{l}^{*}, p\right)\right\} \geq\right.$ 
$D T))$ or $\left(z\left(p_{l}^{*}\right)<\min _{p \in P}\{z(p)\}\right)$, then include the solution $p_{l}^{*}$ into the population $P$. Otherwise, include the random solution $p_{l}$ into the population $P$.

$7 k=k+1$. If $k \leq P S$, then go to Step 2; otherwise, the initial population formation is finished.

Regarding the generation of opposition-based solutions, an easy way to operationalize this is to maintain a long-term frequency array (memory) $f$, where $f(i)$ records the number of times the item $i$ has appeared in a solution. The array $f$ is operated in a very simple way: all one needs is to initialize it with zeros and update its values each time a new solution is generated, i.e., $f\left(p_{l}(i)\right)=f\left(p_{l}(i)\right)+1$, where $p_{l}$ is the currently generated solution. To obtain the opposition-based random solution, it is sufficient to pick up $m$ items with the smallest frequency (ties are broken randomly). Obviously, this ensures that $\delta\left(p_{l}, p_{l-1}\right)=m(l=2,3, \ldots)$, where $p_{l}, p_{l-1}$ are solutions consecutively generated one after another.

Each generated solution is subject to improvement by the hierarchical ITS. After improvement, it is checked if the distance between the improved solution $p_{l}^{*}$ and the population $P\left(\delta\left(p_{l}^{*}, P\right)=\min _{p \in P}\left\{\delta\left(p_{l}^{*}, p\right)\right\}\right)$ is greater than or equal to the pre-defined distance threshold, DT. If it is the case, the improved solution is included into the population. The same is true if the improved solution is better than the best population member. Otherwise, the randomly generated solution enters the population. This ensures both the quality and genetic variance of the initial population.

\subsection{Parent Selection}

At each generation, two solutions (permutations) $p^{\prime}$ and $p^{\prime \prime}$ are randomly selected in the population $P$ to serve as parents for reproduction.

\subsection{Crossover Operator}

The goal of crossover (recombination) operator is to produce an offspring from a pair of parents. The principle of functioning of our crossover is based on two concepts: backbone solution and opposition-based (opposite) solution (see also [19]). This allows both to preserve the common elements (genes) in two selected parents and introduce completely new genes. Note that, in addition, the backbone solution is partially optimized to ensure a higher quality of the off-

\section{Figure 2}

Pseudo-code of the hybrid genetic algorithm

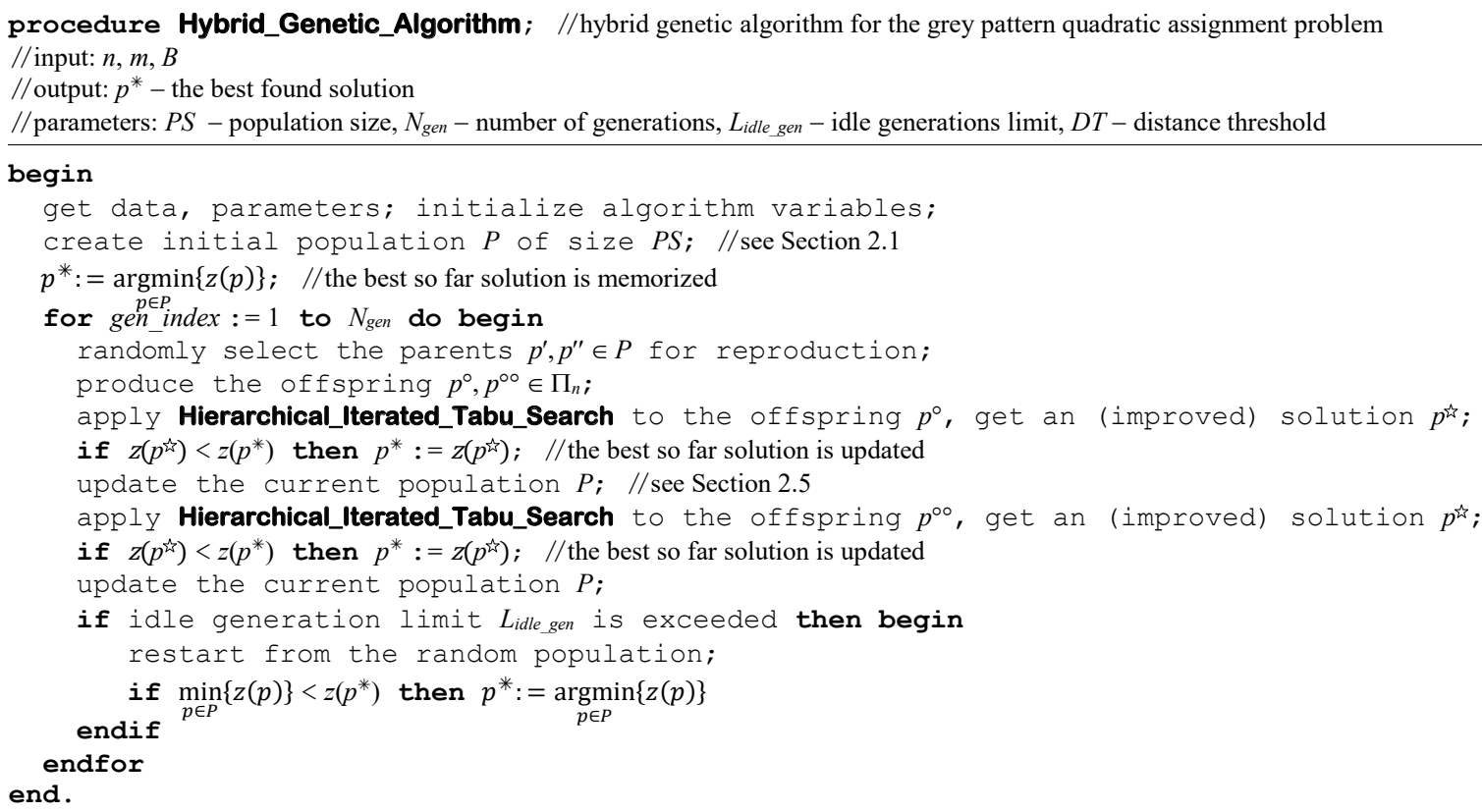


spring. A so-called greedy adaptive procedure (GAP) is applied for this purpose. Thus, two offspring solutions are generated: the optimized offspring solution and its counterpart (the opposite offspring solution). The crossover is called the backbone and opposition-based crossover and its high-level description is given in Figure 3. Some specific details are as follows. The values of the short-term array (gene frequencies) $f_{S T}^{\text {cross }}$ are calculated by this expression:

$$
\begin{aligned}
f_{S T}^{\text {cross }}(i)= & \left|\left\{j: j \in\left\{p^{\prime(k)}: k=1, \ldots, m\right\}, j \in\left\{p^{\prime \prime(k)}: k=1, \ldots, m\right\}\right\}\right|, \\
& i=1, \ldots n
\end{aligned}
$$

where $p^{\prime}, p^{\prime \prime}$ are the corresponding parental solutions. The $m$ genes with the most frequency are then chosen to form the backbone solution $p^{\mathscr{\%}}$. After this, we apply the greedy adaptive procedure, which respects only $m / 2$ genes with the largest frequency. So, the GAP receives a partial solution $p^{\mathscr{\&}}$ (the elements $p^{\mathscr{\&}}(1), \ldots$,

Figure 3

Pseudo-code of the backbone and opposition-based crossover

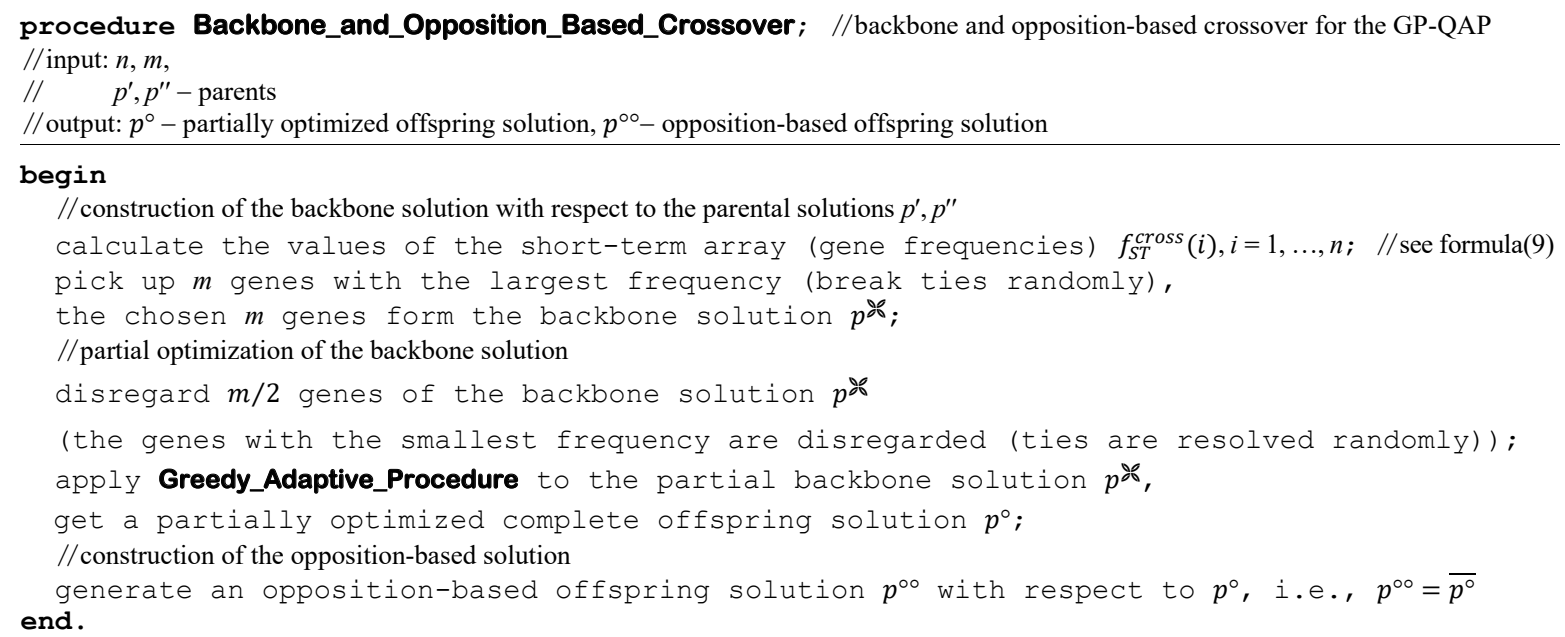

Figure 4

Pseudo-code of the greedy adaptive procedure

procedure Greedy_Adaptive_Procedure; //greedy adaptive procedure for the GP-QAP

//input: $n, m, B$

// $\quad p$ - partial solution, where the elements $p(m / 2+1), \ldots, p(m)$ are disregarded

// output: $p$ - feasible (complete) solution

\section{begin}

for $i:=1$ to $n$ do begin $c(i):=0 ;$ Selected $(i):=$ FALSE endfor;

for $i:=1$ to $n$ do for $j:=1$ to $m / 2-1$ do $c(i):=c(i)+B(i, p(j)) ; / /$ calculation of contributions $(c)$

for $i:=1$ to $m / 2$ do $\operatorname{Selected}(p(i)):=$ TRUE; //initialization of Selected

$i:=m / 2 ; k:=p(m / 2)$;

for $q:=1$ to $m / 2$ do begin //cycle is repeated until the solution has been completed

minimum_contribution $:=\infty$;

for $j:=1$ to $n$ do

if Selected $(j)=$ FALSE then begin

$c(j):=c(j)+B(j, k) ;$ if $c(j)<$ minimum_contribution then begin minimum_contribution $:=c(j) ; j_{\text {min }}:=j$ endif endif;

$i:=i+1 ; p(i):=j_{\min } ; \quad / /$ the element with the minimum contribution is added to the solution

Selected $(p(i)):=$ TRUE; $k:=j_{\min }$

$$
\text { endfor; }
$$

$i:=m+1$; for $j:=1$ to $n$ do $/ /$ assigning values to the elements $p(m+1), \ldots, p(n)$

end.

$$
\text { if } \operatorname{Selected}(j)=\text { FALSE then begin } p(i):=j ; i:=i+1 \text { endif }
$$


$\left.p^{\mathscr{}}(\mathrm{m} / 2)\right)$ as an input. The GAP chooses the element, one at a time, and adds it to the current partial solution. In particular, GAP adds, at each iteration $q(q=$ $1, \ldots, m / 2)$, the element from the set of unselected elements $\{j: j=1, \ldots, n\} \backslash\{p(i): i=1, \ldots, m / 2+q-1\}$ with the minimum contribution value (see formula $(7)$ ) across all the unselected elements, i.e., $\underset{j \in\{j: j=1, \ldots, n\} \backslash\{p(i): i=1, \ldots, m / 2+q-1\}}{j=}\{c(p(j))\}$. This is continued until the solution has been completed (see Figure 4). Note that the objective function value $z$ can be obtained from the $c$ values by the equation: $z=$ $2 \sum_{j=1}^{m} c(p(j))$. The complexity of the GAP algorithm is $O(m n)$.

The principle of greedy adaptive algorithm is not new and it is in its nature similar to "greedy randomized adaptive search procedures" (GRASP) [7]. GAP is adaptive since it selects the current element with respect to the already selected elements and the set of selected elements is updated at every iteration. The greedy component of GAP is that it always chooses the element with the minimum possible contribution. However, randomization is absent in GAP.

For the generation of opposite solution, we utilize a long term frequency array $f_{L T}^{\text {cross }}$. The initialization of $f_{L T}^{\text {cross }}$ is done before running the genetic algorithm. The values of $f_{L T}^{\text {cross }}$ are updated each time the new optimized backbone solution is constructed, i.e., $f_{L T}^{\text {cross }}\left(p^{\circ}(i)\right)=$ $f_{L T}^{c r o s s}\left(p^{\circ}(i)\right)+1$, where $p^{\circ}$ is the optimized backbone solution. To get the opposition-based solution, it is sufficient to pick up $m$ items with the smallest frequency.

\subsection{Improvement of the Offspring: A Hierarchical Iterated Tabu Search Algorithm}

\subsubsection{Hierarchical Iterated Tabu Search Algorithm}

Our proposed hierarchical iterated tabu search algorithm follows the hierarchical iterated local search paradigm [10]. The central idea is that the further enhancement of local search-based algorithms is achieved by intelligently developing the inner structure (architecture) of the algorithms and creating hierarchically structured (hierarchical) algorithms (HAs). The basic principle behind HA is the multiple utilization (reuse) of the well-known heuristic algorithm (like the local search (LS) or tabu search (TS)). In the case of TS, we firstly obtain an iterated tabu search - ITS - by combining tabu search and some perturbations. Further, the ITS algorithm itself is combined with the other ITS algorithm, which re- sults in the "ITS-ITS" algorithm. This can be further iterated. Thus, we can define a hierarchy of many "copies" of the ITS algorithms embedded within each other. Each copy contains the three main ingredients: 1) invocation of the iterated tabu search procedure; 2) candidate acception; 3) perturbation procedure.

\section{A. Tabu search}

The tabu search procedure plays the essential role in the hierarchical ITS algorithm. In the simplest way, the TS procedure uses the 1-exchange neighbourhood $\Theta_{1}$. In particular, TS starts with the current solution and iteratively swaps an element of the set $M=\{p(i): i=1, \ldots, m\}$ with an element of the $\operatorname{set} N=\{p(i): i=m+1, \ldots, n\}$ such that the objective function value is minimized taking into account the tabu condition and aspiration criterion.

To reduce the computational time, we use the modified neighbourhood $\Theta_{1}^{*}$, which is defined as follows (see also [18]):

$$
\begin{aligned}
& \Theta_{1}^{*}(p)=\left\{p^{\prime}: p^{\prime} \in\{p(i): i=1, \ldots, m\} \backslash\right. \\
& \left.\{p(v)\} \cup\{p(w)\}, p(v) \in M^{\prime}, p(w) \in N^{\prime}\right\}
\end{aligned}
$$

where $v=1, \ldots,\left|M^{\prime}\right|, w=m+1, \ldots, m+\left|M^{\prime}\right|$. Sets $M^{\prime}, N^{\prime}$ are formed in the following way:

$$
\begin{aligned}
& M^{\prime}=\left\{p(i): c(p(i)) \geq \text { threshold }_{1}, i=1, \ldots, m\right\}, \\
& N^{\prime}=\left\{p(i): c(p(i)) \leq \text { threshold }_{2}, i=m+1, \ldots, n\right\}
\end{aligned}
$$

where $c$ is the contribution array, threshold $1=$ $\max \{c(p(i)): i=1, \ldots, m\}-\rho B$ Max, $\quad$ threshold $_{2}=$ $\min \{c(p(i)): i=m+1, \ldots, n\}+\rho B M a x, \quad B M a x=$ $\max \left\{b_{k l}: k=1, \ldots n, l=1, \ldots n\right\}, \rho(\rho>0)$ is a parameter (a neighbourhood size factor).

The tabu list TabuList is organized as a matrix, where the tabu list entry TabuList $(p(v), p(w))$ stores the current iteration number plus the tabu tenure $h$, i.e., the number of the iteration starting at which the corresponding elements $(p(v), p(w))$ may again be interchanged. The tabu tenure is fixed at the pre-defined value $(h=$ $\lfloor 0.3 \mathrm{~m} \mid)$. The interchange of elements $p(v), p(w)$ is not allowed if the value of TabuList $(p(v), p(w))$ is equal or greater than the current iteration number. The tabu status is ignored if the aspiration criterion is met, i.e., the interchange results in a solution that is better than the best so far solution. In addition, we disregard the tabu status with a small probability $\alpha,(\alpha=0.02)$ even if the aspiration criterion does not hold. This slightly increases the number of accepted moves and helps avoid- 
ing potential stagnation of the search.

In addition to the tabu list, we also use a long-term memory like mechanism to maintain an archive of good solutions that were evaluated but not chosen [4]. The goal is to diversify the search process and explore more regions of the search space. To implement this mechanism, a list called an archive (Archive) is used, which is composed of so-called "second" solutions. In particular, at each iteration, the best chosen solution becomes the current solution, while the second best solution $p^{\sim}$ is included in Archive. The tabu search restarts from one of these solutions when the best found solution is not improved for $L_{\text {idle_iter }}$ iterations ( $L_{\text {idle_iter }}$ is called an idle iterations limit). In our algorithm, $L_{\text {idle_iter }}=$ $[0.2 \tau\rfloor$, where $\tau$ is the number of tabu search iterations. Note that it is required to save the whole information, i.e., the current solution $p$, the contributions $c$, and the indices of the elements which generate $p^{\sim}$. Archive is emptied after finishing the TS procedure. The pseudocode of the tabu search procedure is shown in Figure 5.

\section{Figure 5}

Pseudo-code of the tabu search algorithm

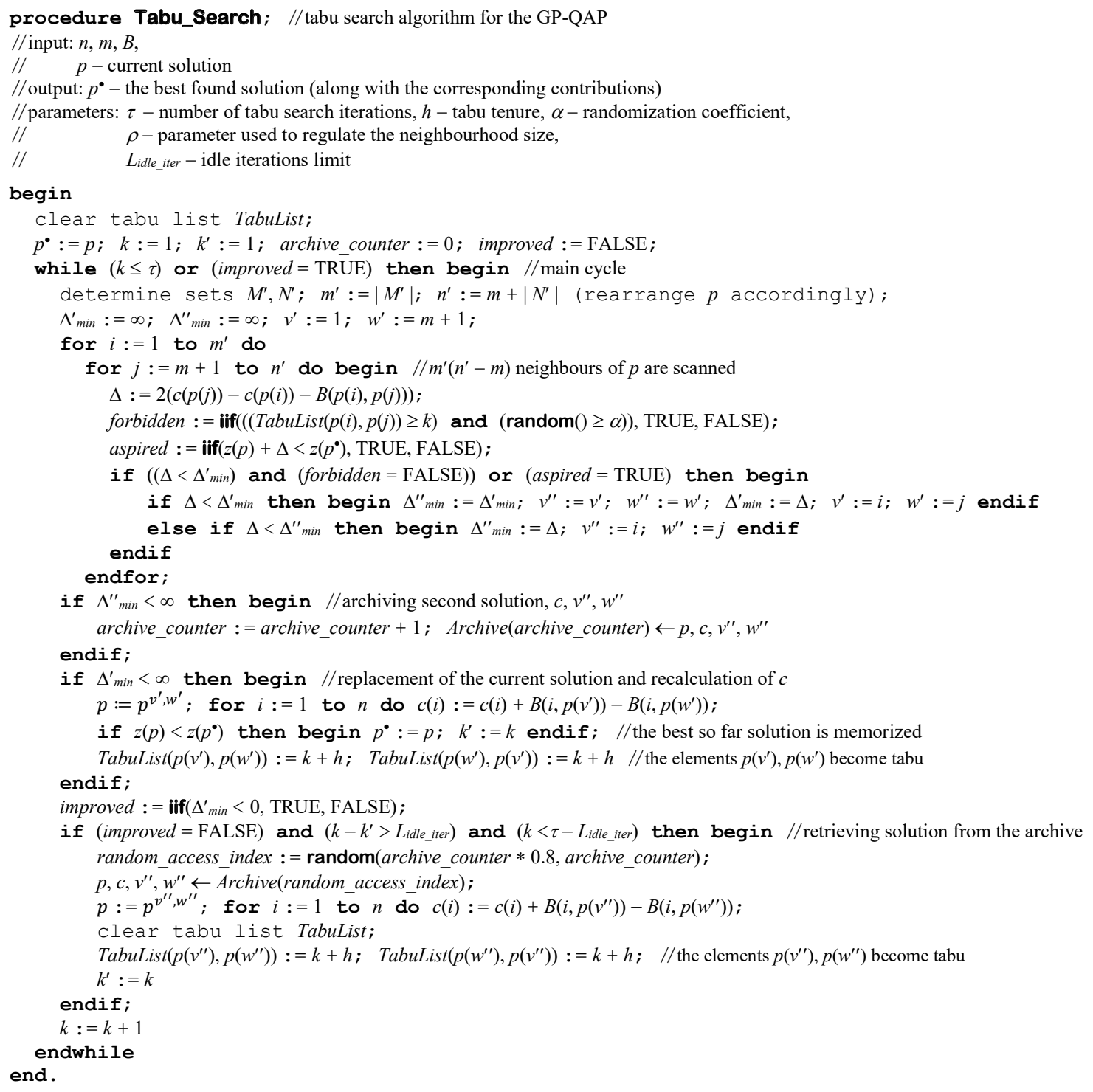

Notes. 1. The immediate if function iif $\left(x, y_{1}, y_{2}\right)$ returns $y_{1}$ if $x=$ TRUE, otherwise it returns $y_{2}$. 2. The function random() returns a pseudorandom number uniformly distributed in $[0,1]$. 3. The function random $\left(x_{1}, x_{2}\right)$ returns a pseudo-random number in $\left[x_{1}, x_{2}\right]$. 


\section{B. Iterated tabu search}

In the iterated tabu search algorithm, the self-contained tabu search described above is combined with some sort of perturbations (see Section 2.4.3). The TS procedure transforms the current solution into the optimized solution. Perturbation is applied to chosen optimized candidate solution that is selected by a defined candidate acception rule (see Section 2.4.2). The perturbed solution serves as an input for the self-contained TS procedure, which starts immediately after the perturbation procedure has been executed. TS again returns an improved solution. This solution (or possibly some other previously optimized solution), in turn, is perturbed, and so on. The best found solu- tion is regarded as the resulting solution of ITS. The overall process continues until a pre-defined number of iterations have been performed (see Figure 6).

\section{Hierarchical iterated tabu search}

The 1-level hierarchical iterated tabu search (1-HITS) algorithm can be obtained from the ITS algorithm. The structure of the algorithm remains practically unchanged, except that the ITS algorithm (instead of the TS algorithm) is used for the solution improvement (see Figure 7).

It is possible to further extend the 1-HITS algorithm in a very gentle way. New extension is entitled as 2 -HITS. The pseudo-code of 2 -HITS is almost identical to

Figure 6

Pseudo-code of the iterated tabu search algorithm

procedure Iterated_Tabu_Search; //iterated tabu search algorithm for the GP-QAP

//input: $p$ - current solution

// output: $p^{\nabla}-$ the best found solution (along with the corresponding contributions)

//parameter: $Q$ - number of iterations

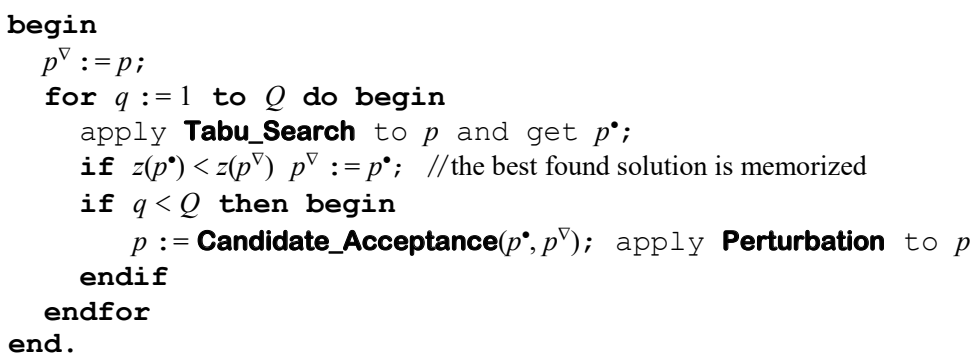

Figure 7

Pseudo-code of the 1-level hierarchical iterated tabu search algorithm

procedure 1-Hierarchical_Iterated_Tabu_Search; //1-level hierarchical iterated tabu search algorithm for the GP-QAP

//input: $p$ - current solution

// output: $p^{\langle 1\rangle}-$ the best found solution (along with the corresponding contributions)

//parameters: $Q_{1}$ - number of iterations

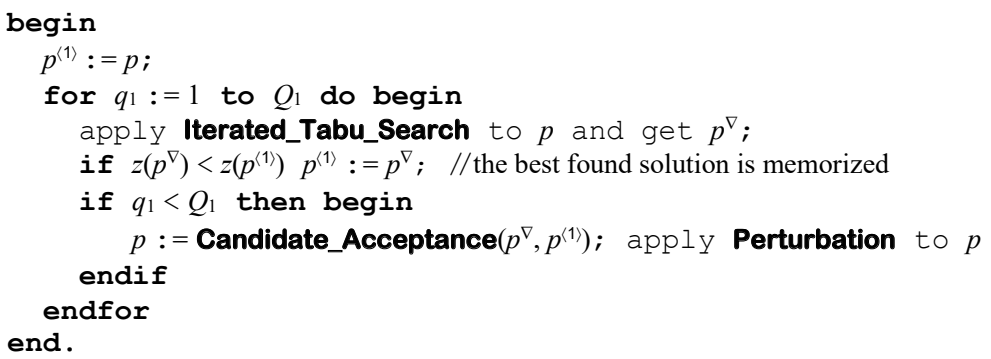


Figure 8

Template of the seven-level hierarchical iterated tabu search algorithm

procedure Hierarchical_Iterated_Tabu_Search; //seven-level hierarchical iterated tabu search for the GP-QAP

//input: $n, m, B$,

// $\quad p$-current solution

//output: $p$ - the best found solution

//parameters: $Q, Q_{1}, \ldots, Q_{7}$ - numbers of iterations

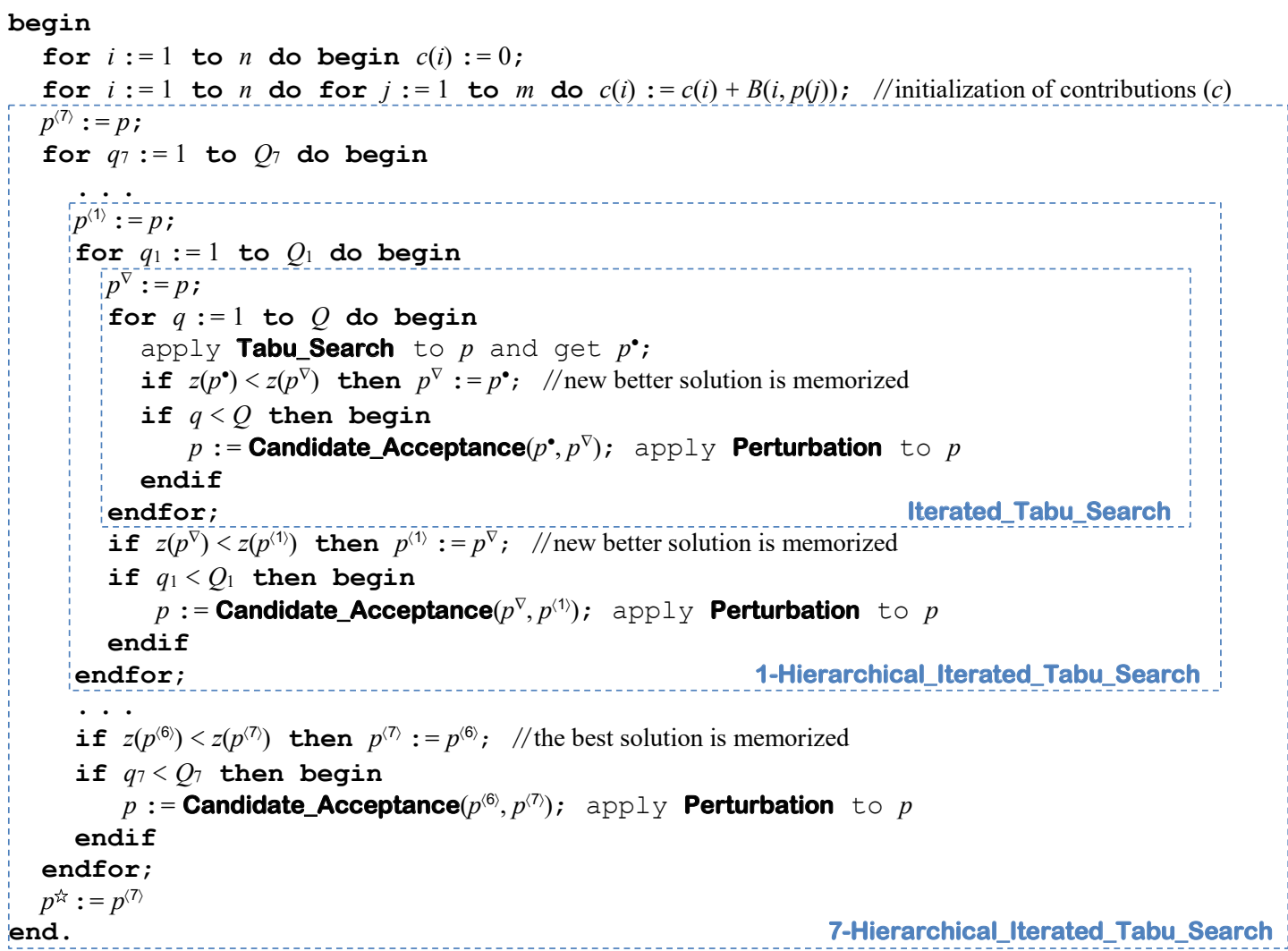

the one of 1-HITS, except that the invocation of the ITS procedure is substituted by the invocation of the 1-HITS procedure. Continuing in the above manner, one can create a cascade of self-similar algorithms: 3-HITS, 4-HITS, and so on. It is not difficult, only one should be careful and patient. Our most latest version of HITS is, in particular, 7-HITS. The description of 7-HITS is presented in Figure 8. It is a high level template of the algorithm, rather than a detailed pseudo-code. Some parts are omitted for the sake of brevity.

\subsubsection{Candidate Acception}

The function Candidate_Acceptance can be imple- mented in many different ways. We utilize the socalled "where-you-are" rule, which means that we always choose the first candidate from the function parameters' list. For example, in the case of Candidate_Acceptance $\left(p^{*}, p^{\Delta}\right), p^{\bullet}$ is accepted.

\subsubsection{Perturbation}

The perturbation procedure is very simple in its structure and it consists of two parts: a) random mutation (shuffling) and b) re-construction of the mutated solution by fast greedy adaptive procedure (see Figure 9).

Firstly, the accepted candidate solution undergoes a random mutation process; in particular, the solution is 
"disintegrated" by disregarding (removing) $\mu$ elements from the current solution ( $\mu$ is a parameter called mutation rate). The $\mu$ elements are chosen in a random way (see Figure 10). The value of $\mu$ is relatively small in our algorithm $(\mu=[0.15 \mathrm{~m}])$, so only a minor fraction of elements is involved in the mutation procedure.

Secondly, the mutated partial solution is subject to re-construction (partial optimization) by the fast greedy adaptive procedure (FGAP), which is identical to that used in the crossover operator, except that the more effective calculation of the contributions is applied (see Figure 11). The calculation takes $O(\mu n)$ time. The overall complexity of the FGAP algorithm is also $O(\mu n)$. This results in a very fast execution of both FGAP and HITS as long as the value of $\mu$ is not large.

\subsection{Population Management}

After the offspring is improved by HITS, it is tested if the new solution ( $p$ ) differs from the other solutions in population. If it is the case, it is checked if the new solution is better than the best solution in the population or the distance between the new solution and population $\left(\delta(p, P)=\min _{p \in P}\{\delta(p, p)\}\right)$ is greater than or equal to the distance threshold $D T$. If this is true, then the new solution replaces the worst solution in the current population $\left(P=P \cup\{p\} \backslash\left\{p_{\text {worst }}\right\}\right.$, where $p_{\text {worst }}=\operatorname{argmax}_{p \in P}\{z(p)\}$ ). (Otherwise, the population remains unaltered and the algorithm continues with the next generation.) This rule is to maintain both the high-quality and sufficient diversity of the members of population.

\section{Figure 9}

Pseudo-code of the perturbation procedure

procedure Perturbation; //perturbation procedure for the GP-QAP

//input: $p$ - current solution

// output: $p$ - the resulting (perturbed) solution

//parameter: $\mu$ - mutation rate (number of disregarded elements)

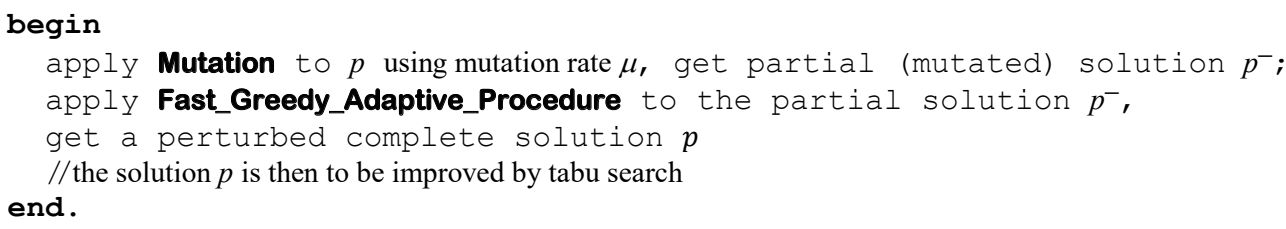

Figure 10

Pseudo-code of the random mutation procedure

procedure Mutation; //random mutation procedure for the GP-QAP

//input: $m$,

// $\quad p$-current (complete) solution

// output: $p^{-}-$resulting (partial) solution

\section{begin}

for $i:=1$ to $m-1$ do begin //shuffling the items $p(1), \ldots, p(m)$ generate an integer $j$ randomly, uniformly, $i \leq j \leq m$;

$p:=p^{i, j} \quad / /$ the items $p(i)$ and $p(j)$ are interchanged

\section{endfor;}

$/ /$ after shuffling, the items $p(m-\mu+1), \ldots, p(m)$ are disregarded (no action is needed)

$p^{-}:=p$

end. 


\section{Figure 11}

Pseudo-code of the fast greedy adaptive procedure

procedure Fast_Greedy_Adaptive_Procedure ; //fast greedy adaptive procedure for the GP-QAP

//input: $n, m, B$,

// $\quad p-$ partial solution, where the elements $p(m-\mu+1), \ldots, p(m)$ are disregarded

//output: $p$ - feasible (complete) solution

//parameter: $\mu$ - mutation rate $(1 \leq \mu<m)$

\section{begin}

for $i:=1$ to $n$ do for $j:=m-\mu+1$ to $m$ do $c(i):=c(i)-B(i, p(j))$; //fast (re)calculation of contributions $(c)$

for $i:=1$ to $n$ do $\operatorname{Selected}(i):=$ FALSE; for $i:=1$ to $m-\mu$ do Selected $(p(i)):=$ TRUE; //initialization of Selected

$i:=m-\mu ; \quad k:=p(m-\mu)$;

for $q:=1$ to $\mu$ do begin //cycle is repeated until the solution has been completed

minimum_contribution $:=\infty$;

for $j:=1$ to $n$ do

if Selected $(j)=$ FALSE then begin

$c(j):=c(j)+B(j, k) ;$ if $c(j)<$ minimum contribution then begin minimum contribution $:=c(j) ; j_{\min }:=j$ endif endif;

$i:=i+1 ; p(i):=j_{\min } ; \quad / /$ the element with the minimum contribution is added to the solution

$\operatorname{Selected}(p(i)):=$ TRUE; $k:=j_{\text {min }}$

endfor;

$i:=m+1$; for $j:=1$ to $n$ do if $\operatorname{Selected}(j)=$ FALSE then begin $p(i):=j ; i:=i+1$ endif end.

\subsection{Restart}

The restart of the genetic algorithm takes place if the solutions of the population are not improved for $L_{i \text { - }}$ dle_gen generations ( $L_{\text {idle_gen }}$ is an idle generations limit, which is set to $\left[0.15 N_{\text {gen }}\right], N_{\text {gen }}$ is the number of generations). The restart is performed by simply constructing the new population (see Section 2.1).

\section{Results of Computational Experiments}

Our new hybrid genetic algorithm (NHGA) was implemented by using C\# programming language. The computational experiments have been carried out on a $3 \mathrm{GHz}$ personal computer running Windows 7 Enterprise.

We have tested our algorithm on the medium and large-scaled GP-QAP instances with $n=256$ and $n=$ 1024, respectively. The instances are generated according to the method described in $[15]^{3}$. The grids

3 These instances can also be found at the website: http://www. personalas.ktu.lt/ alfmise/. are of dimensions $16 \times 16\left(n_{1}=n_{2}=16\right)$ and $32 \times 32\left(n_{1}=\right.$ $\left.n_{2}=32\right)$, respectively. The grey density parameter $m$ varies from 2 to 128 and from 2 to 512 .

The values of the control parameters of NHGA used in the experiments are shown in Table 1. (Note that the calibration of the parameters was not performed.) Firstly, we have experimented with the problems of size 256 and we have compared our algorithm with the improved genetic-evolutionary algorithm (IGEA) presented in [12]. To our knowledge, IGEA seems very likely to be the most efficient (to date) heuristic algorithm for the problems of this size. As the algorithms IGEA and NHGA constantly find the best known (pseudo-optimal) solutions (BKSs), we compare the run time performance, rather than the quality of solutions.

Thus, the experimentation was designed in such a way that the algorithms IGEA and NHGA were run 10 and 100 times, respectively. At every run, the algorithms are applied to a given $m$, each time starting from new random solutions. The current run is finished as soon as BKS has been found (even without 
Table 1

Values of the control parameters of the hybrid genetic algorithm

\begin{tabular}{l|l|l}
\multicolumn{1}{c|}{ Parameter } & \multicolumn{1}{c}{ Value } & \multicolumn{1}{c}{ Remarks } \\
\hline Population size, $P S$ & 20 & \\
\hline Number of generations, $N_{\text {gen }}$ & 40 & $0<L_{\text {idle_gen }} \leq N_{\text {gen }}$ \\
\hline Idle generations limit, $L_{\text {idlegen }}$ & $\left\lfloor 0.15 N_{\text {gen }}\right\rfloor$ & $0 \leq D T \leq m$ \\
\hline Distance threshold, $D T$ & $\lfloor 0.25 \mathrm{~m}\rfloor$ & $Q_{H I E R}=Q \times Q_{1} \times Q_{2} \times Q_{3} \times Q_{4} \times Q_{5} \times Q_{6} \times Q_{7}{ }^{\dagger}$ \\
\hline Number of hierarchical tabu search iterations, $Q_{H I E R}$ & 384 & \\
\hline Number of tabu search iterations, $\tau$ & 80 & $h>0$ \\
\hline Tabu tenure, $h$ & $\lfloor 0.3 m\rfloor$ & $0<L_{\text {idle_iter }} \leq \tau$ \\
\hline Idle iterations limit, $L_{\text {idle_iter }}$ & $\lfloor 0.2 \tau\rfloor$ & $\rho>0$ \\
\hline Neighbourhood size factor, $\rho$ & 0.4 & $0<\alpha<1$ \\
\hline Randomization coefficient, $\alpha$ & 0.02 & $0<\mu<m$ \\
\hline Mutation rate, $\mu$ & $\lfloor 0.15 \mathrm{~m}\rfloor$ & \\
\hline
\end{tabular}

${ }^{\dagger} Q=Q_{1}=Q_{2}=Q_{3}=Q_{4}=Q_{5}=Q_{6}=2, Q_{7}=3$

reaching the limit of generations $\left.N_{\text {gen }}\right)$. The obtained run times (CPU times) of the algorithms to achieve the BKS for every $m$ are reported in Table 2. For the algorithm IGEA, the run time averaged over 10 runs is presented. For the algorithm NHGA, the run time of the shortest run out of 100 runs is given. The comparison thus seems to be slightly unfair - we just wanted to bring to the light how fast our algorithm can run.

On the whole, the results demonstrate that NHGA clearly dominates IGEA. IGEA was able to slightly outperform NHGA in very few cases only $(m=26,101,102,103)$. Figure 12 illustrates the incredible overall speed improvement of NHGA for $m^{\prime}$ s varying from 30 to 100 . It can be observed that, for some instances, the computation time is reduced by a factor of over 100 (!). Such small run times of NHGA indicate that NHGA is capable to obtain pseudo-optimal solutions at very early stages of the construction of the initial population with the help of the stand-alone hierarchical tabu search only. Very probably, the efficiency of NHGA could be improved even more by an accurate tuning of the values of the control parameters.

During the additional extensive, long-lasting experimentation, we were examining the algorithm NGHA on the large-sized problems ( $n=1024)$, which are much more difficult and time-consuming. It should be stressed that, nevertheless, we were successful in discovering new record breaking solutions for more than 190 values of $m$. The results are presented in Table 3. The CPU times are omitted. The new BKVs are in bold face. All the remaining values are from [13].

The further thorough experiments are needed to show (verify) the pseudo-optimality of the newly obtained best known solutions.

We also provide several visual representations (grey frames) corresponding to some of the new best known solutions (in particular, $m=401,402,403,404,405,40$ $6,407,408$ ) (see Figure 13). In the graphical illustrations, each

1024-square-grid is replicated 8 times horizontally and 8 times vertically for the visibility convenience. 
Table 2

Results of the experiments with the medium-sized GP-QAP instances $(n=256)$

\begin{tabular}{|c|c|c|c|c|c|c|c|c|c|c|c|c|c|c|}
\hline \multirow{2}{*}{$m$} & \multirow{2}{*}{$\begin{array}{l}\text { Best known } \\
\text { value (BKV) }\end{array}$} & \multirow{2}{*}{$\begin{array}{l}\text { Dev. } \\
\text { from } \\
\text { BKV }\end{array}$} & \multicolumn{2}{|c|}{ CPU time (sec) } & \multirow{2}{*}{$m$} & \multirow{2}{*}{$\begin{array}{c}\text { Best known } \\
\text { value } \\
(\mathrm{BKV})\end{array}$} & \multirow{2}{*}{$\begin{array}{l}\text { Dev. } \\
\text { from } \\
\text { BKV }\end{array}$} & \multicolumn{2}{|c|}{ CPU time (sec) } & \multirow{2}{*}{$m$} & \multirow{2}{*}{$\begin{array}{l}\text { Best known } \\
\text { value (BKV) }\end{array}$} & \multirow{2}{*}{$\begin{array}{l}\text { Dev. } \\
\text { from } \\
\text { BKV }\end{array}$} & \multicolumn{2}{|c|}{ CPU time (sec) } \\
\hline & & & IGEA & NHGA & & & & IGEA & NHGA & & & & IGEA & NHGA \\
\hline 2 & 1562 & 0 & 0.0 & 0.00 & 45 & 8674910 & 0 & 150 & 5.91 & 87 & 39389054 & 0 & 25.0 & 0.19 \\
\hline 3 & 7810 & 0 & 0.0 & 0.00 & 46 & 9129192 & 0 & 64 & 11.38 & 88 & 40416536 & 0 & 23.0 & 0.15 \\
\hline 4 & 15620 & 0 & 0.0 & 0.00 & 47 & 9575736 & 0 & 3.1 & 0.29 & 89 & 41512742 & 0 & 183 & 6.98 \\
\hline 5 & 38072 & 0 & 0.0 & 0.00 & 48 & 10016256 & 0 & 2.0 & 0.16 & 90 & 42597626 & 0 & 165 & 4.76 \\
\hline 6 & 63508 & 0 & 0.0 & 0.00 & 49 & 10518838 & 0 & 3.4 & 0.56 & 91 & 436764774 & 0 & 224 & 17.64 \\
\hline 7 & 97178 & 0 & 0.0 & 0.00 & 50 & 11017342 & 0 & 2.8 & 0.56 & 92 & 44759294 & 0 & 157 & 7.94 \\
\hline 8 & 131240 & 0 & 0.0 & 0.00 & 51 & 11516840 & 0 & 7.5 & 0.83 & 93 & 45870244 & 0 & 214 & 19.18 \\
\hline 9 & 183744 & 0 & 0.0 & 0.00 & 52 & 12018388 & 0 & 6.3 & 0.39 & 94 & 46975856 & 0 & 190 & 21.52 \\
\hline 10 & 242266 & 0 & 0.0 & 0.00 & 53 & 12558226 & 0 & 4.6 & 0.54 & 95 & 48081112 & 0 & 169 & 2.14 \\
\hline 11 & 304722 & 0 & 0.1 & 0.00 & 54 & 13096646 & 0 & 4.0 & 0.03 & 96 & 49182368 & 0 & 216 & 6.58 \\
\hline 12 & 368952 & 0 & 0.1 & 0.00 & 55 & 13661614 & 0 & 10.1 & 0.12 & 97 & 50344050 & 0 & 213 & 23.88 \\
\hline 13 & $45^{r} 7504$ & 0 & 0.1 & 0.00 & 56 & 14229492 & 0 & 2.8 & 0.15 & 98 & 51486642 & 0 & 188 & 63.40 \\
\hline 14 & 547522 & 0 & 0.1 & 0.00 & 57 & 14793682 & 0 & 2.2 & 0.37 & 99 & 52660116 & 0 & 201 & 50.53 \\
\hline 15 & 644036 & 0 & 0.1 & 0.00 & 58 & 15363628 & 0 & 2.3 & 0.09 & 100 & 53838088 & 0 & 117 & 48.18 \\
\hline 16 & 742480 & 0 & 0.1 & 0.00 & 59 & 15981086 & 0 & 3.5 & 0.71 & 101 & 55014262 & 0 & 84 & 156 \\
\hline 17 & 878888 & 0 & 0.2 & 0.00 & 60 & 16575644 & 0 & 2.4 & 0.95 & 102 & 56202826 & 0 & 40.0 & 115 \\
\hline 18 & 1012990 & 0 & 0.1 & 0.00 & 61 & 17194812 & 0 & 2.2 & 0.01 & 103 & 57417112 & 0 & 73 & 84.00 \\
\hline 19 & 1157992 & 0 & 0.2 & 0.00 & 62 & 17822806 & 0 & 3.6 & 0.01 & 104 & 58625240 & 0 & 62 & 51.14 \\
\hline 20 & 1305744 & 0 & 0.3 & 0.08 & 63 & 18435790 & 0 & 1.9 & 0.00 & 105 & 59854744 & 0 & 38.0 & 32.41 \\
\hline 21 & 1466210 & 0 & 0.5 & 0.00 & 64 & 19050432 & 0 & 2.3 & 0.00 & 106 & 61084902 & 0 & 33.0 & 10.85 \\
\hline 22 & 1637794 & 0 & 0.3 & 0.00 & 65 & 19848790 & 0 & 3.1 & 0.00 & 107 & 62324634 & 0 & 21.0 & 0.73 \\
\hline 23 & 1820052 & 0 & 0.2 & 0.00 & 66 & 20648754 & 0 & 4.5 & 0.02 & 108 & 63582416 & 0 & 12.6 & 0.65 \\
\hline 24 & 2010846 & 0 & 0.6 & 0.02 & 67 & 21439396 & 0 & 9.7 & 0.08 & 109 & 64851966 & 0 & 11.1 & 1.02 \\
\hline 25 & 2215714 & 0 & 3.2 & 0.31 & 68 & 22234020 & 0 & 18.0 & 0.23 & 110 & 66120434 & 0 & 10.7 & 0.41 \\
\hline 26 & 2426298 & 0 & 16.5 & 22.98 & 69 & 23049732 & 0 & 27.0 & 0.64 & 111 & 67392724 & 0 & 8.2 & 0.46 \\
\hline 27 & 2645436 & 0 & 1.1 & 0.02 & 70 & 23852796 & 0 & 26.0 & 0.98 & 112 & 68666416 & 0 & 7.7 & 0.17 \\
\hline 28 & 2871704 & 0 & 0.9 & 0.03 & 71 & 24693608 & 0 & 78 & 0.43 & 113 & 69984758 & 0 & 10.2 & 0.13 \\
\hline 29 & 3122510 & 0 & 0.7 & 0.03 & 72 & 25522408 & 0 & 490 & 64.80 & 114 & 71304194 & 0 & 6.3 & 0.18 \\
\hline 30 & 3373854 & 0 & 0.5 & 0.00 & 73 & 26375828 & 0 & 298 & 5.81 & 115 & 72630764 & 0 & 5.1 & 0.37 \\
\hline 31 & 3646344 & 0 & 0.6 & 0.00 & 74 & 27235240 & 0 & 304 & 2.50 & 116 & 73962220 & 0 & 5.3 & 0.21 \\
\hline 32 & 3899744 & 0 & 0.5 & 0.02 & 75 & 28114952 & 0 & 41.0 & 0.85 & 117 & 75307424 & 0 & 4.0 & 0.03 \\
\hline 33 & 4230950 & 0 & 0.7 & 0.03 & 76 & 29000908 & 0 & 121 & 1.30 & 118 & 76657014 & 0 & 3.6 & 0.06 \\
\hline 34 & 4560162 & 0 & 2.6 & 0.36 & 77 & 29894452 & 0 & 145 & 4.81 & 119 & 78015914 & 0 & 2.3 & 0.03 \\
\hline 35 & 4890132 & 0 & 3.2 & 0.41 & 78 & 30797954 & 0 & 117 & 1.15 & 120 & 79375832 & 0 & 1.7 & 0.05 \\
\hline 36 & 5222296 & 0 & 2.0 & 0.44 & 79 & 31702182 & 0 & 11.6 & 0.81 & 121 & 80756852 & 0 & 1.6 & 0.07 \\
\hline 37 & 5565236 & 0 & 1.8 & 0.34 & 80 & 32593088 & 0 & 3.3 & 0.47 & 122 & 82138768 & 0 & 1.4 & 0.03 \\
\hline 38 & 5909202 & 0 & 0.9 & 0.14 & 81 & 33544628 & 0 & 3.9 & 0.26 & 123 & 83528554 & 0 & 1.0 & 0.04 \\
\hline 39 & 6262248 & 0 & 1.1 & 0.08 & 82 & 34492592 & 0 & 70 & 3.33 & 124 & 84920540 & 0 & 0.7 & 0.01 \\
\hline 40 & 6613472 & 0 & 0.9 & 0.02 & 83 & 35443938 & 0 & $5 \% .0$ & 1.41 & 125 & 86327812 & 0 & 0.4 & 0.00 \\
\hline 41 & 7002794 & 0 & 0.6 & 0.11 & 84 & 36395172 & 0 & 61 & 2.77 & 126 & 87736646 & 0 & 0.3 & 0.00 \\
\hline 42 & 7390586 & 0 & 0.7 & 0.16 & 85 & 37378800 & 0 & 151 & 1.20 & 127 & 89150166 & 0 & 0.2 & 0.00 \\
\hline 43 & 7794422 & 0 & 3.2 & 0.20 & 86 & 38376438 & 0 & 94 & 0.32 & 128 & 90565248 & 0 & 0.2 & 0.00 \\
\hline 44 & 8217264 & 0 & 16.0 & 0.87 & & & & & & & & & & \\
\hline
\end{tabular}

The deviation from BKV (Dev. from BKV) is calculated as the ratio $\left(z^{*}-B K V\right) / B K V$, where $z^{*}$ denotes the algorithms' best found solution. The best known values of the objective function corresponding to the best known solutions are from [12]. 
Table 3

Results of the experiments with the large-sized GP-QAP instances $(n=1024)$

\begin{tabular}{|c|c|c|c|c|c|c|c|c|c|c|c|c|c|c|c|}
\hline$m$ & BKV & $m$ & BKV & $m$ & BKV & $m$ & BKV & $m$ & BKV & $m$ & BKV & $m$ & BKV & $m$ & BKV \\
\hline 2 & 390 & 66 & 5132250 & 130 & 23460170 & 194 & 57086766 & 258 & 106260632 & 322 & 176518174 & 386 & 262819150 & 450 & 363665156 \\
\hline 3 & 1954 & 67 & 5312762 & 131 & 23897592 & 195 & 57739124 & 259 & 107273354 & 323 & 177756358 & 387 & 264317294 & 451 & 365361310 \\
\hline 4 & 3908 & 68 & 5493398 & 132 & 24335656 & 196 & 58392270 & 260 & 108286116 & 324 & 178959204 & 388 & $265^{\prime \prime 9} 91714$ & 452 & 367056444 \\
\hline 5 & 9488 & 69 & 5675784 & 133 & 24773832 & 197 & 59055298 & 261 & 109299348 & 325 & 180158842 & 389 & 267231448 & 453 & 368754728 \\
\hline 6 & 15882 & 70 & 5868614 & 134 & 25213730 & 198 & 59710314 & 262 & 110313464 & 326 & 181377560 & 390 & 268698768 & 454 & 370451410 \\
\hline 7 & 24290 & 71 & 6061636 & 135 & 25653062 & 199 & 60357328 & 263 & 111323160 & 327 & 182598340 & 391 & 270205824 & 455 & 372157104 \\
\hline 8 & 32808 & 72 & 6253544 & 136 & 26091040 & 200 & 61005880 & 264 & 112349256 & 328 & 183826382 & 392 & 271688930 & 456 & 373863658 \\
\hline 9 & 45844 & 73 & 6451748 & 137 & 26536474 & 201 & 61656140 & 265 & 113366358 & 329 & 185039942 & 393 & 273203922 & 457 & 375575430 \\
\hline 10 & 60310 & 74 & 6658646 & 138 & 26980064 & 202 & 62313154 & 266 & 114381534 & 330 & 186245648 & 394 & 274660438 & 458 & 377289052 \\
\hline 11 & 75878 & 75 & 6866464 & 139 & 27426740 & 203 & 62979360 & 267 & 115403394 & 331 & 187480294 & 395 & 276180114 & 459 & 379005274 \\
\hline 13 & 114040 & 77 & 7287952 & 141 & 28319430 & 205 & 64329116 & 269 & 117454596 & 333 & 189934056 & 397 & 2791729'78 & 461 & 382449898 \\
\hline 14 & 136706 & 78 & 7497962 & 142 & 28761578 & 206 & 65021762 & 270 & 118462682 & 334 & 191134986 & 398 & 280677404 & 462 & $3841^{174962}$ \\
\hline 15 & 160770 & 79 & r7708934 & 143 & 29211334 & 207 & 65721964 & 271 & 119472414 & 335 & 192370030 & 399 & 282179032 & 463 & 385902750 \\
\hline 16 & 185552 & 80 & ry919112 & 144 & 29649520 & 208 & 66422364 & 272 & 120505424 & 336 & $1935965^{r 76}$ & 400 & 283712492 & 464 & 387628048 \\
\hline 17 & 218392 & 81 & 8147012 & 145 & 30118164 & 209 & 67136312 & 273 & $1215^{r} 745^{r} 72$ & 337 & 194844496 & 401 & 285211916 & 465 & 389368514 \\
\hline 18 & 251618 & 82 & 8363950 & 146 & 30588480 & 210 & 67852496 & 274 & 122629730 & 338 & 196104848 & 402 & 286746076 & 466 & 391105794 \\
\hline 19 & 288006 & 83 & 8600584 & 147 & 31065948 & 211 & 68585494 & 275 & 123626322 & 339 & $19^{r} 7349^{r} 714$ & 403 & 288238662 & 467 & 392843892 \\
\hline 20 & 324794 & 84 & 8839620 & 148 & 31546098 & 212 & 69315338 & 276 & 124716364 & 340 & 198600114 & 404 & $289^{r} 784468$ & 468 & 394587900 \\
\hline 21 & 365546 & 85 & 9079818 & 149 & 32025690 & 213 & 70050648 & 277 & $125^{\prime \prime} 741472$ & 341 & 199870596 & 405 & 291260654 & 469 & 396332818 \\
\hline 22 & 407406 & 86 & 9322672 & 150 & 32508848 & 214 & ro789536 & 278 & 126807112 & 342 & 201181102 & 406 & 292833208 & 470 & 398083462 \\
\hline 23 & 451448 & 87 & 9563920 & 151 & 32992712 & 215 & r71527862 & 279 & 127842588 & 343 & 202519622 & 4077 & 294387042 & 471 & 399837320 \\
\hline 26 & 603368 & 90 & 10331422 & 154 & 34461110 & 218 & 73726832 & 282 & 1310 r77338 & 346 & 206544390 & 410 & 298998414 & 474 & 405112104 \\
\hline 27 & 659044 & 91 & 10600710 & 155 & 34955468 & 219 & 74466810 & 283 & 132132040 & 347 & 207925194 & 411 & 300564200 & 475 & 406875344 \\
\hline 28 & 716280 & 92 & 10871062 & 156 & 35450196 & 220 & 75201458 & 284 & $13318^{r} 7012$ & 348 & 209234904 & 412 & 302129606 & 476 & 408637620 \\
\hline 29 & r777436 & 93 & 11138470 & 157 & 35944108 & 221 & 75953890 & 285 & $13428^{\prime 7484}$ & 349 & 210612178 & 413 & 303688980 & 477 & 410409038 \\
\hline 30 & 837798 & 94 & 11411510 & 158 & 36437606 & 222 & 76713866 & 286 & 135364426 & 350 & 211922934 & 414 & 305224788 & 478 & 412182568 \\
\hline 31 & 907090 & 95 & 11679880 & 159 & 36933614 & 223 & rary65610 & 287 & 136441394 & 351 & 213304876 & 415 & 306845268 & 479 & 413959340 \\
\hline 32 & 975008 & 96 & 11944352 & 160 & 37426912 & 224 & r78218352 & 288 & 137549224 & 352 & 214686716 & 416 & 308393388 & 480 & 415733856 \\
\hline 33 & 1050792 & 97 & 12237102 & 161 & 37947342 & 225 & 78977922 & 289 & 138637260 & 353 & 216044260 & 417 & 309969764 & 481 & 417519180 \\
\hline 34 & 1125558 & 98 & 12523996 & 162 & 38464394 & 226 & 79744456 & 290 & 139677068 & 354 & $21^{r} 73^{r} 65^{r} 74$ & 418 & 311420318 & 482 & 419302686 \\
\hline 35 & 1203646 & 99 & 12813836 & 163 & 38982592 & 227 & 80520900 & 291 & $1408012^{r} 72$ & 355 & $218^{r} 738658$ & 419 & 313094242 & 483 & 421092758 \\
\hline 36 & 1281132 & 100 & 13103420 & 164 & 39500208 & 228 & 81287994 & 292 & 141875610 & 356 & 220109066 & 420 & 314652760 & 484 & 422883164 \\
\hline 37 & 1368444 & 101 & 13398254 & 165 & 40025416 & 229 & 82061894 & 293 & 142916356 & 357 & 221526988 & 421 & 316172166 & 485 & 424678088 \\
\hline 38 & 1456842 & 102 & 13691306 & 166 & 40550006 & 230 & 82837128 & 294 & 144026694 & 358 & 222888300 & 422 & 317825280 & 486 & 426473544 \\
\hline 39 & 1547598 & 103 & 13988062 & 167 & $410^{\prime \prime} 8930$ & 231 & 83613898 & 295 & 145175322 & 359 & 224268836 & 423 & 319428868 & 487 & 428272184 \\
\hline 40 & 1638808 & 104 & 14288780 & 168 & 41606240 & 232 & 84406568 & 296 & 146290048 & 360 & 225646838 & 424 & 321022500 & 488 & 430071632 \\
\hline 41 & 1736236 & 105 & 14593444 & 169 & 42140968 & 233 & 85225404 & 297 & 147454448 & 361 & 227020504 & 425 & 322637088 & 489 & 431876322 \\
\hline 42 & 1834074 & 106 & 14899130 & 170 & 42673974 & 234 & 86030804 & 298 & 148527002 & 362 & 228390592 & 426 & 324266210 & 490 & 433683572 \\
\hline 43 & 1935946 & 107 & 15216394 & 171 & 43219476 & 235 & 86829778 & 299 & 149672540 & 363 & 229827232 & 427 & 325898680 & 491 & 435492454 \\
\hline 44 & 2042792 & 108 & 15537796 & 172 & 43787404 & 236 & 87618540 & 300 & 150827224 & 364 & 231201722 & 428 & $32745^{r} 7994$ & 492 & 437303524 \\
\hline
\end{tabular}


Table 3 (continued)

\begin{tabular}{|c|c|c|c|c|c|c|c|c|c|c|c|c|c|c|c|}
\hline$m$ & BKV & $m$ & BKV & $m$ & BKV & $m$ & BKV & $m$ & BKV & $m$ & BKV & $m$ & BKV & $m$ & BKV \\
\hline 45 & 2147200 & 109 & $1585^{\prime 7934}$ & 173 & 44361196 & 237 & 88445972 & 301 & 151952048 & 365 & 232578308 & 429 & $3289932 \% 0$ & 493 & 439118116 \\
\hline 46 & 2260650 & 110 & 161777106 & 174 & 44933388 & 238 & 89239062 & 302 & 153122860 & 366 & 233960416 & 430 & 330616714 & 494 & 440933678 \\
\hline 47 & 2373506 & 111 & 16504524 & 175 & 45511224 & 239 & 90075414 & 303 & 154282700 & 367 & 235302078 & 431 & 332246332 & 495 & 442752278 \\
\hline 48 & 2482832 & 112 & 16837956 & 176 & 46091442 & 240 & 90875504 & 304 & $15541^{17} 120$ & 368 & 236712932 & 432 & 333874768 & 496 & $4445^{r} 70032$ \\
\hline 49 & 2607474 & 113 & 17174378 & 177 & 46680202 & 241 & 91698908 & 305 & 156547208 & 369 & 238200964 & 433 & 335514106 & 497 & 446397066 \\
\hline 50 & 2730510 & 114 & 17508602 & 178 & 47274350 & 242 & 92523578 & 306 & $15^{r} 7684310$ & 370 & 239582944 & 434 & 337154026 & 498 & 448224550 \\
\hline 51 & 2857088 & 115 & 17849756 & 179 & 47871440 & 243 & 93371894 & 307 & 158836480 & 371 & 241044336 & 435 & 338796402 & 499 & 450053654 \\
\hline 52 & 2988998 & 116 & 18191920 & 180 & 48462430 & 244 & 94187252 & 308 & 159999648 & 372 & 242479798 & 436 & 340435998 & 500 & 451883116 \\
\hline 53 & 3120248 & 117 & 18535442 & 181 & 49056670 & 245 & 95044544 & 309 & 161157378 & 373 & 243893396 & 437 & 342076542 & 501 & 453718668 \\
\hline 54 & 3257234 & 118 & 18902942 & 182 & 49654614 & 246 & 95865322 & 310 & 162344014 & 374 & 245361204 & 438 & 343718980 & 502 & 455554546 \\
\hline 56 & 3535048 & 120 & 19631156 & 184 & 50876864 & 248 & 97531736 & 312 & 164641724 & 376 & 248246874 & 440 & 347009592 & 504 & 459230104 \\
\hline 57 & $36844^{r} 78$ & 121 & 20001764 & 185 & 51494526 & 249 & 98361638 & 313 & 165838280 & 377 & 249710800 & 441 & 348669786 & 505 & $4610^{\prime 73188}$ \\
\hline 58 & 3829950 & 122 & 20370638 & 186 & 52115066 & 250 & 99225594 & 314 & 167015058 & 378 & 251141622 & 442 & 350325606 & 506 & 462916382 \\
\hline 59 & 3984538 & 123 & 20746696 & 187 & 52731636 & 251 & 100062350 & 315 & 168180928 & 379 & 252535872 & 443 & 351981700 & 507 & 464761614 \\
\hline 60 & 4136400 & 124 & 21117234 & 188 & 53348334 & 252 & 100898116 & 316 & 169354960 & 380 & 254011358 & 444 & 353638456 & 508 & 466607612 \\
\hline 61 & 4291962 & 125 & 21484868 & 189 & 53959660 & 253 & 101733670 & 317 & 170529852 & 381 & 255486362 & 445 & 355296090 & 509 & 468457260 \\
\hline 62 & 4447434 & 126 & 21852518 & 190 & $545^{r 71808}$ & 254 & 102566006 & 318 & 171723964 & 382 & 256914322 & 446 & 356954184 & 510 & 470307298 \\
\hline 63 & 4604860 & 127 & 22218924 & 191 & 55185346 & 255 & 103399158 & 319 & $172910^{\prime \prime} 68$ & 383 & 258421702 & 447 & 358612252 & 511 & 472158510 \\
\hline 64 & 4762688 & 128 & 22581376 & 192 & 55788864 & 256 & 104232704 & 320 & 174113066 & 384 & 259861698 & 448 & 360270272 & 512 & 474010112 \\
\hline 65 & 4949042 & 129 & 23021790 & 193 & 56452088 & 257 & 105247082 & 321 & 175343494 & 385 & 261377866 & 449 & 361968220 & & \\
\hline
\end{tabular}

Figure 12

Illustration of the run time improvement. In the y-axis, we present the ratio of $\mathrm{CPU}_{\mathrm{IGEA}}$ to $\mathrm{CPU}_{\mathrm{NHGA}}$, where $\mathrm{CPUI}_{\mathrm{GEA}}, \mathrm{CPU}_{\mathrm{NHGA}}$ denote the run times of IGEA and NHGA, respectively

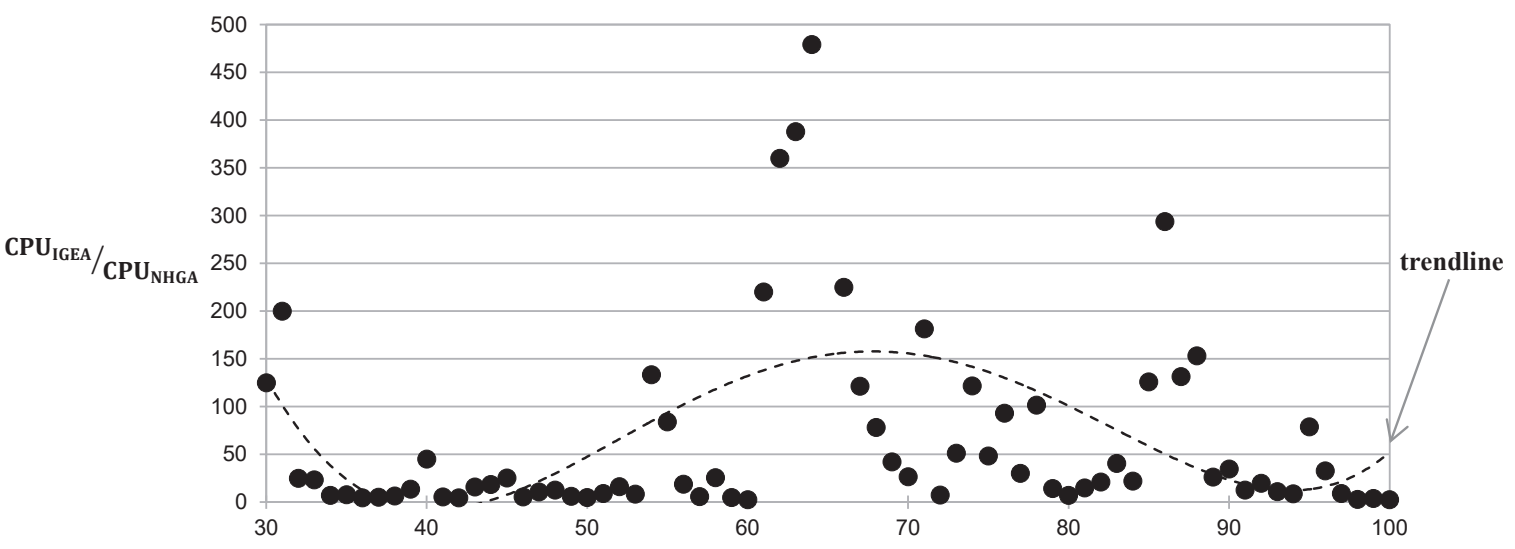

Grey density parameter, $m$ 


\section{Figure 13}

Examples of (pseudo-)optimal grey frames $(n=1024)$ : (a) $m=4$ 01, (b) $m=4$ 02, (c) $m=403$, (d) $m=404$, (e) $m=405$, (f) $m=406$, (g) $m=407$, (h) $m=408$

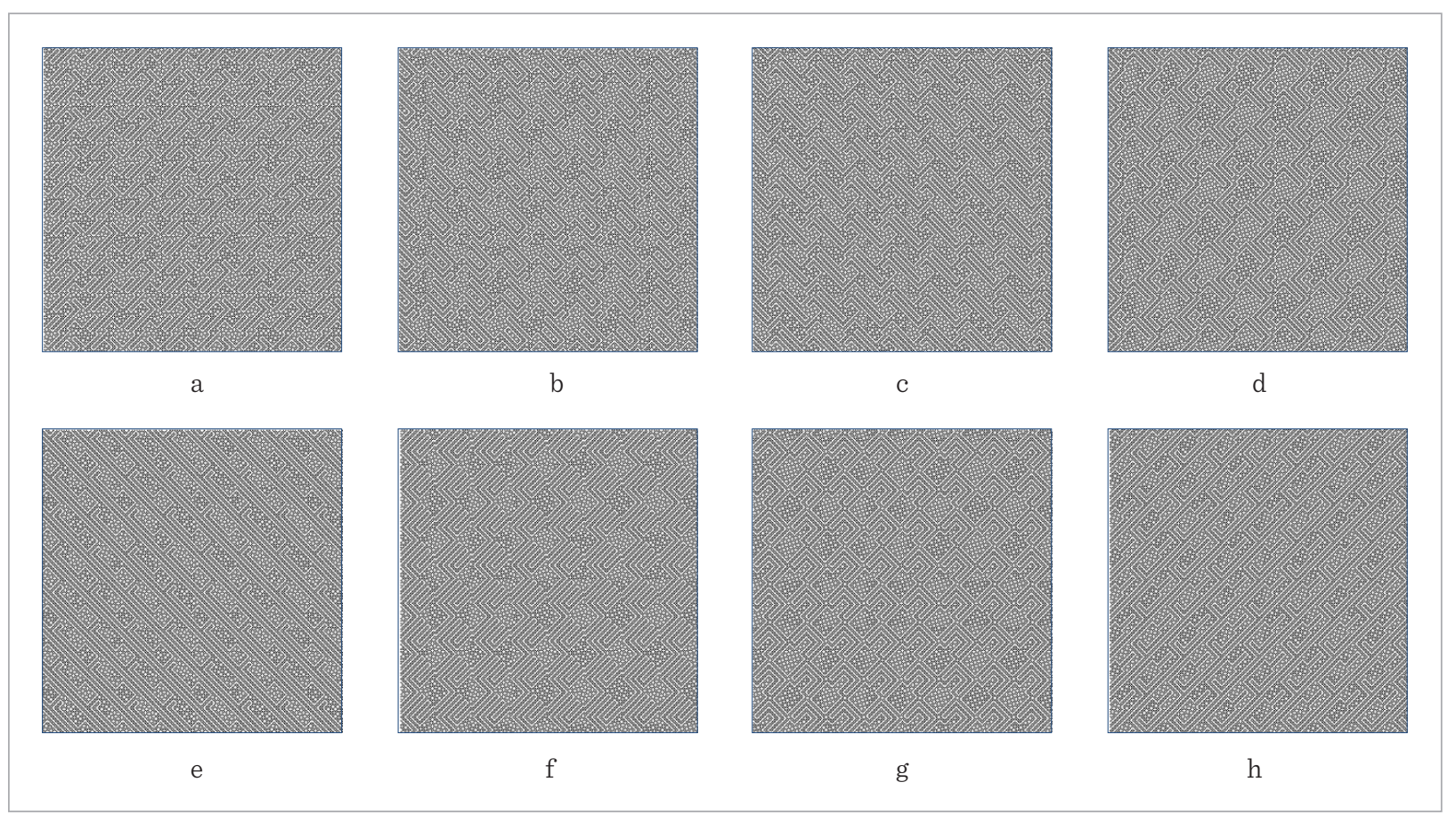

\section{Concluding Remarks}

In this paper, we have proposed a new improved hybrid genetic algorithm for solving the grey pattern quadratic assignment problem. In particular, the genetic algorithm is hybridized with the hierarchical iterated tabu search and this is adopted for the GP-QAP for the first time. The compacted, reduced neighbourhood is used. This enables very fast execution of the hierarchical ITS algorithm and the hybrid GA. In addition, we apply a smart combination of the iterated tabu search and the greedy adaptive perturbations. This allows beneficial balance between diversification and intensification during the iterative search process.

Our algorithm has been computationally tested on the medium and large-sized instances of the GP-QAP, where the instances size is equal to 256 and 1024, respectively. The results obtained from the conducted experiments demonstrate that the new hybridized GA is extremely effective and outperforms other the-state-of-the-art heuristic algorithms. The high efficiency is confirmed by the numerous best known solutions achieved for the challenging GP-QAP instances of size 1024.

Regarding the future work, it might be worthy to improve the population initialization and management mechanisms within hybrid GA to avoid presumable stagnation of the genetic process. It is also worth to try to further enhance the performance of HGA by developing new architecture of the genetic algorithm itself.

Additionally, our proposed new HGA might be adapted for other types of combinatorial optimization problems. 


\section{References}

1. Aarts, E. H. L., Lenstra, J.K. (Eds.). Local Search in Combinatorial Optimization, Wiley, 1997.

2. Çela,E.The Quadratic AssignmentProblem: Theory and Algorithms, Kluwer, 1998. https://doi.org/10.1007/9781-4757-2787-6

3. Daskin, M. Network and Discrete Location: Models, Algorithms and Applications, John Wiley, 1995. https:// doi.org/10.1002/9781118032343

4. Dell'Amico, M., Trubian, M. Solution of Large Weighted Equicut Problems. European Journal of Operational Research, 1998, 106(2-3), 500-521. https://doi. org/10.1016/S037r7-2217(97)00287-7

5. Drezner, Z. Finding a Custer of Points and the Grey Pattern Quadratic Assignment Problem. OR Spectrum, 2006, 28(3), 417-436. https://doi.org/10.1007/s00291005-0010-7

6. Drezner, Z., Misevičius, A., Palubeckis, G. Exact Algorithms for the Solution of the Grey Pattern Quadratic Assignment Problem. Mathematical Methods of Operations Research, 2015, 82(1), 85-105. https://doi. org/10.1007/s00186-015-0505-1

7. Feo, T. A., Resende, M. G. C. Greedy Randomized Adaptive Search Procedures. Journal of Global Optimization, 1995, 6(2), 109-133. https://doi.org/10.1007/BF01096763

8. Glover, F., Laguna, M. Tabu Search, Kluwer, 1997. https://doi.org/10.1007/978-1-4615-6089-0

9. Goldberg, D. E. Genetic Algorithms in Search, Optimization and Machine Learning. Addison-Wesley, 1989.

10. Hussin, M. S., Stützle, T. Hierarchical Iterated Local Search for the Quadratic Assignment Problem. In: Blesa, M. J., Blum, C., Di Gaspero, L., Roli, A., Sampels, M., Schaerf, A. (Eds.), Hybrid Metaheuristics, HM 2009, Lecture Notes in Computer Science, Springer, 2009, 5818, 115-129. https://doi.org/10.1007/978-3-642-04918-7_9

11. Misevičius, A. Experiments with Hybrid Genetic Algorithm for the Grey Pattern Problem. Informatica, 2006, $17(2), 237-258$.
12. Misevičius, A. Generation of Grey Patterns Using an Improved Genetic-Evolutionary Algorithm: Some New Results. Information Technology and Control, 2011, 40(4), 330-343. https://doi.org/10.5755/j01.itc.40.4.983

13. Misevičius, A., Guogis, E., Stanevičienè, E. Computational Algorithmic Generation of High-Quality Colour Patterns. In: Skersys, T., Butleris, R., Butkienè, R. (Eds.), Information and Software Technologies (ICIST 2013), Communications in Computer and Information Science, Springer, Berlin, Heidelberg, 2013, 403, 285-296. https://doi.org/10.1007/978-3-642-41947-8_24

14. Misevičius, A., Rubliauskas, D. Performance of Hybrid Genetic Algorithm for the Grey Pattern Problem. Information Technology and Control, 2005, 34(1), 15-24.

15. Taillard, E. Comparison of Iterative Searches for the Quadratic Assignment Problem. Location Science, 1995, 3(2), 87-105. https://doi.org/10.1016/09668349(95)00008-6

16. Taillard, E., Gambardella, L. M. Adaptive Memories for the Quadratic Assignment Problem. Tech. Report IDSIA-87-97, Lugano, Switzerland, 1997.

17. Tizhoosh, H. R. Opposition-Based Learning: A New Scheme for Machine Intelligence. In: Mohammadian, M. (Ed.), Proceedings of International Conference on Computational Intelligence for Modeling, Control and Automation and International Conference on Intelligent Agents, Web Technologies and Internet Commerce (CIMCA/IAWTIC), IEEE Press, 2005, 2, 695701. https://doi.org/10.1109/CIMCA.2005.1631345

18. Wu, Q., Hao, J.-K. A Hybrid Metaheuristic Method for the Maximum Diversity Problem. European Journal of Operational Research, 2013, 231(2), 452-464. https:// doi.org/10.1016/j.ejor.2013.06.002

19. Zhou, Y., Hao, J.-K., Duval, B. Opposition-Based Memetic Search for the Maximum Diversity Problem. IEEE Transactions on Evolutionary Computation, 2017,21(5), 731-745. https://doi.org/10.1109/TEVC.2017.2674800 